\title{
Multimodal transfer of MDR by exosomes in human osteosarcoma
}

\author{
ELENA TORREGGIANI $^{1}$, LAURA RONCUZZI $^{1}$, FRANCESCA PERUT $^{1}$, \\ NICOLETTA ZINI ${ }^{2,3}$ and NICOLA BALDINI ${ }^{1,4}$ \\ ${ }^{1}$ Laboratory for Orthopaedic Pathophysiology and Regenerative Medicine, Istituto Ortopedico Rizzoli, \\ I-40136 Bologna; ${ }^{2}$ Laboratory of Musculoskeletal Cell Biology, Istituto Ortopedico Rizzoli, \\ I-40136 Bologna; ${ }^{3} \mathrm{CNR}$ - National Research Council of Italy, Institute of Molecular Genetics, \\ I-40129 Bologna; ${ }^{4}$ Department of Biomedical and Neuromotor Sciences, \\ University of Bologna, I-40136 Bologna, Italy
}

Received January 8, 2016; Accepted February 22, 2016

DOI: $10.3892 /$ ijo.2016.3509

\begin{abstract}
Exosomes are extracellular vesicles released by both normal and tumour cells which are involved in a new intercellular communication pathway by delivering cargo (e.g., proteins, microRNAs, mRNAs) to recipient cells. Tumour-derived exosomes have been shown to play critical roles in different stages of tumour growth and progression. In this study, we investigated the potential role of exosomes to transfer the multidrug resistance (MDR) phenotype in human osteosarcoma cells. Exosomes were isolated by differential centrifugation of culture media from multidrug resistant human osteosarcoma MG-63DXR30 (Exo/DXR) and MG-63 parental cells (Exo/S). Exosome purity was examined by transmission electron microscopy and confirmed by immunoblot analysis for the expression of specific exosomal markers. Our data showed that exosomes derived from doxorubicin-resistant osteosarcoma cells could be taken up into secondary cells and induce a doxorubicin-resistant phenotype. The incubation of osteosarcoma cells with Exo/DXR decreased the sensitivity of parental cells to doxorubicin, while exposure with Exo/S was ineffective. In addition, we demonstrated that Exo/DXR expressed higher levels of MDR-1 mRNA and P-glycoprotein compared to Exo/S ( $\mathrm{p}=0.03)$. Interestingly, both MDR-1 mRNA and P-gp increased in MG-63 cells after incubation with Exo/DXR, suggesting this as the main mechanism of exosome-mediated transfer of drug resistance. Our findings suggest that multidrug resistant osteosarcoma cells are able to spread their ability to resist the effects of doxorubicin treat-
\end{abstract}

Correspondence to: Dr Laura Roncuzzi, Laboratory for Orthopaedic Pathophysiology and Regenerative Medicine, Istituto Ortopedico Rizzoli, Via di Barbiano 1/10, I-40136 Bologna, Italy

E-mail: laura.roncuzzi@ior.it

Key words: exosomes, drug-resistance, $\mathrm{P}$-glycoprotein, doxorubicin, osteosarcoma ment on sensitive cells by transferring exosomes carrying MDR-1 mRNA and its product P-glycoprotein.

\section{Introduction}

Exosomes are small RNA and protein containing extracellular vesicles that are able to mediate hetero- and homotypic intercellular communication (1). These natural nanovectors are formed through inward budding of endosomal membranes, giving rise to intracellular multivesicular bodies that integrate into the plasma membrane, and are eventually released (2). Different cell types have been shown to produce exosomes of biologic significance into the extracellular space and the biologic fluids, including B cells, dendritric cells, T cells, platelets, stem cells, and cancer cells (3-8). We have recently demonstrated a role of exosomes as mediators of platelet lysate activity in bone regeneration through the ability to influence osteogenic differentiation and promote cell proliferation and the migration of mesenchymal stromal cell (9). In cancer, exosomes can facilitate tumour progression by supplying the tumour niche with molecules that favour the progression of oncogenic processes, such as proliferation, invasion and metastasis, modulation of immune response, and drug resistance (10-12). Recently, Corcoran et al (13) have demonstrated the ability of prostate cancer cells to transfer multidrug resistance (MDR) phenotype via microvesicles/exosomes, suggesting that, in the context of tumour microenvironment, the development of the MDR phenotype can be mediated, at least in part, by the transfer of P-glycoprotein (P-gp) and microRNAs by exosomes $(13,14)$. Similar results have been obtained in breast cancer, where the transfer of P-gp and miRNAs by exosomes modulates cell cycle distribution and drug-induced apoptosis $(15,16)$.

Osteosarcoma (OS) is the most common primary bone cancer in children and adolescents. Despite aggressive treatment regimens, the outcome is unsatisfactory, particularly in patients with metastatic and/or recurrent disease (17). Treatment failure is commonly due to the development of chemoresistance (18), which appears to be mediated by a variety of mechanisms (19). 
In this study we investigated the potential role of exosomes to transfer a drug resistance phenotype in human OS cells. For this purpose we purified and characterized exosomes from an MDR OS cell line (20) and we showed, for the first time, that exosomes transfer functional MDR-1 mRNA and its product $\mathrm{P}$-glycoprotein to drug-sensitive cells in vitro. This intercellular transfer provides an additional pathway for the cellular acquisition and dissemination of drug-resistant traits indicating exosomes as important mediators in the spread of MDR in human osteosarcoma.

\section{Materials and methods}

Drug. A stock solution of doxorubicin (DXR) (Sigma-Aldrich, Milan, Italy) was prepared in dimethylsulfoxide $(5 \mathrm{mg} / \mathrm{ml})$ and stored at $-20^{\circ} \mathrm{C}$. Appropriate concentrations of DXR solution were freshly diluted before each experiment.

Cell culture. The human OS cell line MG-63 was obtained from the American Type Culture Collection and validated in May 2014 by short-tandem repeat profiling of extracted genomic DNA generated by ATCC-LGC standards. MDR cell line MG-63DXR30 was established from the parental MG-63 (20). Cells were grown in Iscove's modified Dulbecco's medium (IMDM) (Gibco, Thermo Fisher Scientific, Waltham, MA, USA), supplemented with $10 \%$ heat inactivated fetal bovine serum (FBS) (Sigma-Aldrich), $100 \mathrm{U} / \mathrm{ml}$ penicillin, and $0.1 \mathrm{mg} / \mathrm{ml}$ streptomycin (Lonza, Milan, Italy). Drug-resistant variant MG-63DXR30, was continuously cultured in the presence of the selective drug concentration $(30 \mathrm{ng} / \mathrm{ml}$ doxorubicin). All cell lines were maintained at $37^{\circ} \mathrm{C}$ in a humidified $5 \% \mathrm{CO}_{2}$ atmosphere.

Co-culture assays. The effect of MG-63DXR30 secretome on MG-63 viability was evaluated by the Boyden chamber assay with $0.4-\mu \mathrm{m}$ pore membrane filters (Costar, Corning Inc., NY, USA). MG-63 or MG-63DXR30 cells were seeded $\left(5 \times 10^{3}\right.$ cells/well) into the upper chamber, and MG-63 cells were applied at equal proportion to the lower chamber. Twenty-four hours after seeding, $10 \mathrm{ng} / \mathrm{ml}$ of doxorubicin was added to the lower chamber. After $72 \mathrm{~h}$, cell viability was assessed using the Alamar Blue assay (Invitrogen, Thermo Fisher Scientific). The fluorescence was read at 535-590 nm using a microplate-reader (Tecan Infinite F200pro, Tecan, Milan, Italy). The results were expressed as relative fluorescence units (RFU).

Exosome isolation andpurification.MG-63 and MG-63DXR30 cells were cultured until $70 \%$ confluence. Cells were washed with phosphate-buffered saline (PBS) and incubated for two consecutive periods with IMDM supplemented with $10 \%$ FBS depleted of exosomes (FDE) obtained via ultracentrifugation (9). Following collection of the supernatant, the exosomes were concentrated by differential centrifugation: $500 \mathrm{x} g$ for $10 \mathrm{~min}$ (two times), 2,000 x g for $15 \mathrm{~min}$ (two times), and $10,000 \times \mathrm{g}$ for $30 \mathrm{~min}$ (two times) at $4^{\circ} \mathrm{C}$ to remove floating cells and cellular debris. The supernatant was then ultracentrifuged at $110,000 \mathrm{xg}$ for $1 \mathrm{~h}$ at $4^{\circ} \mathrm{C}$. The exosome pellet was resuspended in PBS and centrifuged at 110,000 $\mathrm{x}$ g for $1 \mathrm{~h}$ at $4^{\circ} \mathrm{C}$ (Beckman Coulter, Milan, Italy). The exosome pellet was re-suspended in PBS and stored at $-80^{\circ} \mathrm{C}$ until use. Exosome quantity was determined by the Bradford method (Bio-Rad, Milan, Italy). The exosomes extracted from the supernatant of MG-63 and from the medium of MG-63DXR30 were named Exo/S and Exo/DXR, respectively.

Electron microscopy. Exosomes were resuspended in $2 \%$ paraformaldehyde (PFA) and loaded onto formvar/carboncoated grids. Next, exosomes were fixed in $1 \%$ glutaraldehyde, washed, and counterstained with a solution of uranyloxalate, $\mathrm{pH} 7.0$, embedded in a mixture of $4 \%$ uranylacetate and 2\% methylcellulose before observation with a Zeiss-EM 109 electron microscope (Zeiss, Jena, Germany).

Western blot analysis. Exosomes and cell pellets were treated with RIPA lysis buffer (1\% Triton X-100, 10\% Na-deoxycholate, $5 \mathrm{M} \mathrm{NaCl}, 1 \mathrm{M}$ Tris-HCl pH 7.4, 0.5 M EGTA pH 8.0, $1 \mathrm{M}$ $\mathrm{NaF}$ ) and protease inhibitor cocktail (Roche, Milan, Italy) for $20 \mathrm{~min}$ at $4^{\circ} \mathrm{C}$. Nuclei and cell debris were removed by centrifugation. The protein concentration was determined using the Bradford assay (Bio-Rad). The total cellular proteins and exosomal proteins were resolved by $10 \%$ SDS-polyacrylamide gel and transferred to a nitrocellulose membrane (Thermo Fisher Scientific). The membrane was blocked with $5 \%$ bovine serum albumin (BSA) (Sigma-Aldrich) in T-TBS (0.1 M Tris- $\mathrm{HCl} \mathrm{pH} \mathrm{8.0,} \mathrm{1.5} \mathrm{M} \mathrm{NaCl} \mathrm{and} \mathrm{1 \%} \mathrm{Tween-20)} \mathrm{for} 1 \mathrm{~h}$ at room temperature. Subsequently, the membranes were incubated with rabbit polyclonal CD63 (sc-15363) (1:500), mouse polyclonal calnexin (sc-23954) (1:500) and mouse polyclonal MDR-1/P-gp (sc-55510) (1:200) (Santa Cruz Biotechnology, Santa Cruz, CA, USA) antibodies overnight at $4^{\circ} \mathrm{C}$. After vigorous washing in T-TBS, the membranes were incubated with the secondary antibody for $1 \mathrm{~h}$ at room temperature. Antirabbit antibody (NA934VS) (1:1,000) for CD63, anti-mouse antibody (NA931VS) $(1: 2,000)$ for calnexin and MDR-1/P-gp (GE Healthcare, Milan, Italy), all conjugated to horseradish peroxidase were diluted in T-TBS containing 5\% BSA and used as secondary antibodies. Immunocomplexes were detected with the ECL western blot analysis system (Amersham Pharmacia Biotech, Piscataway, NJ, USA). Reversible Ponceau S (SigmaAldrich) staining was used to assess equal gel loading.

Exosome labelling and uptake. Exosomes were labelled using the PKH26 Red Fluorescent Cell Linker kit (SigmaAldrich) according to the manufacturer's instructions with minor modifications (9). Two microgram ( $2 \mu \mathrm{g})$ of the PKH26 labelled exosomes, or the same volume of the PKH26-PBS control, were resuspended in IMDM supplemented with $10 \%$ FDE and added to $9 \times 10^{3} \mathrm{MG}-63$ cells maintained at $37^{\circ} \mathrm{C}$ in a humidified atmosphere with $5 \% \mathrm{CO}_{2}$. All samples were ultracentrifuged at $110,000 \mathrm{x}$ g for $1 \mathrm{~h}$ at $4^{\circ} \mathrm{C}$ before being added to the cells. After 4 and $24 \mathrm{~h}$ of incubation, uptake was stopped by washing and fixation in $3.7 \%$ PFA for $10 \mathrm{~min}$. Cells were then stained with a fluorescein isothiocyanate (FITC)conjugated phalloidin (Sigma-Aldrich) and visualised with a Nikon Eclipse E800M fluorescence microscope (Nikon, Tokyo, Japan). All cells per high power-field were counted, and the percentage of PKH26 positively stained cells was determined. Five representative high power-fields per sample were evaluated. 


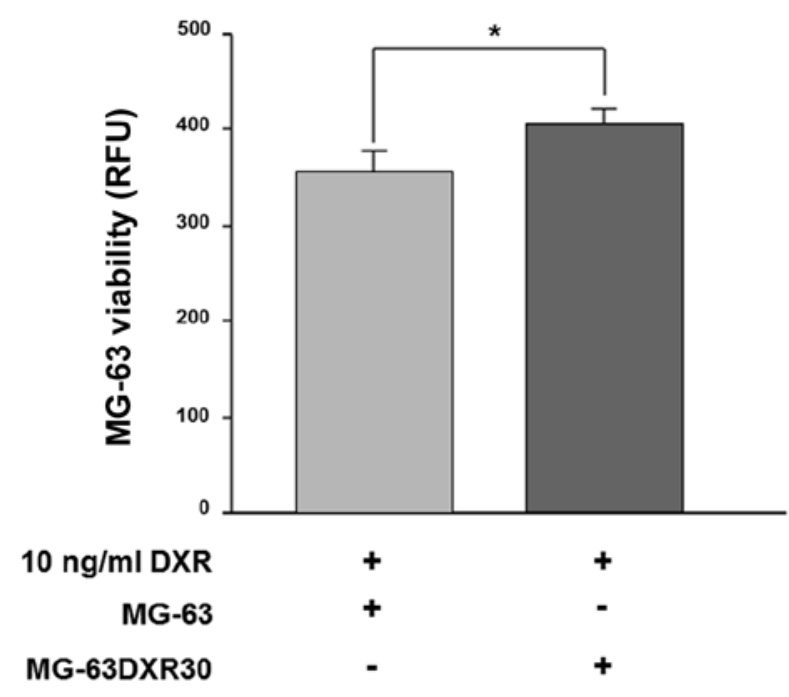

Figure 1. MG-63 cell viability after co-culture with MG-63DXR30. MG-63 co-cultured with MG-63DXR30 showed significantly higher viability than the control. Results represent duplicate biological repeats, each including three technical repeats and are displayed as mean of the relative fluorescence units $(\mathrm{RFU}) \pm \mathrm{SD}$, where ${ }^{*} \mathrm{p}<0.05$. DXR, doxorubicin.

Cell viability. Cell viability was evaluated by the acid phosphatase assay (Sigma-Aldrich) as previously described (21). MG-63 cells were seeded in 96-well, flat-bottomed tissue culture plates at a density of $2 \times 10^{3}$ cells/well. After $24 \mathrm{~h}$, cells were treated with $1.5 \mu \mathrm{g}$ of Exo/S or Exo/DXR or PBS (control), in the presence of IMDM + 10\% FDE. Four hours after exosome addition, cells were treated with increasing concentrations of doxorubicin $(1-60 \mathrm{ng} / \mathrm{ml})$ for $72 \mathrm{~h}$. Briefly, the cells were washed and incubated at $37^{\circ} \mathrm{C}$ with $100 \mu 1$ of buffer containing $0.1 \mathrm{M}$ sodium acetate $\mathrm{pH}$ 5.0, 0.1\% Triton X-100, and $5 \mathrm{mM}$ p-nitrophenil phosphate. After $2 \mathrm{~h}$, the reaction was stopped with the addition of $10 \mu \mathrm{l}$ of $1 \mathrm{~N} \mathrm{NaOH}$, and colour development was assayed at $405 \mathrm{~nm}$ using a microplate reader (Tecan Infinite F200pro). Data are reported as cell survival in respect to untreated cells (set $=100 \%$ ). All experiments were performed three times in triplicate.

Wound-healing assay. Confluent MG-63 and MG-63DXR30 cell monolayers were scratched with a sterile $100-\mu 1$ pipette tip and incubated with or without Exo/S or Exo/DXR. Cell migration was monitored for $24 \mathrm{~h}$ under a Nikon Eclipse-TE 2000-S microscope (Nikon). The widths of the 'wound' (scratched areas) were measured by the NIS-Elements Image Software BR 4.00.00 (Nikon) and the proportion of wound healing was calculated by the following formula: $100 \%$ (width after $24 \mathrm{~h} /$ width at the beginning) x 100\%.

Quantitative real-time polymerase chain reaction ( $q R T$-PCR). Cells were seeded in 12 -well plates $\left(1.5 \times 10^{4}\right.$ cells/well $)$. The following day, cells were treated with $7.5 \mu \mathrm{g}$ of Exo/S or Exo/DXR or PBS (control) for $72 \mathrm{~h}$. Total RNA from cells and exosomes $(n=3)$ was extracted using TRIzol $^{\circledR}$ reagent (Invitrogen, Life Technologies, Monza, Italy) (9). To confirm that the RNA was confined to exosomes, Exo/S and Exo/DXR were treated with $0.4 \mu \mathrm{g} / \mu \mathrm{l}$ RNase A (Sigma-Aldrich) for $1 \mathrm{~h}$ at $37^{\circ} \mathrm{C}$. Total mRNA was reverse transcribed using the MULV
A
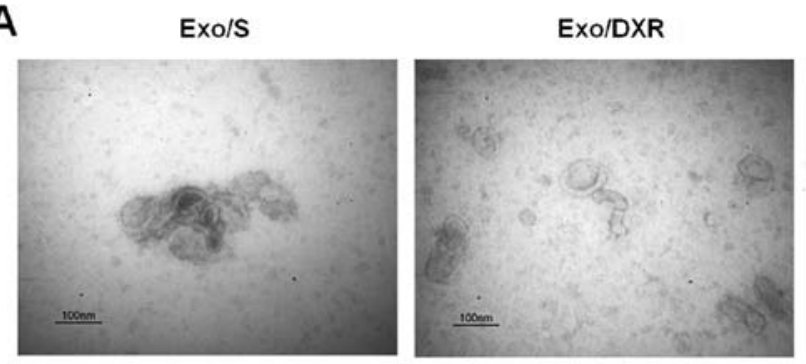

B
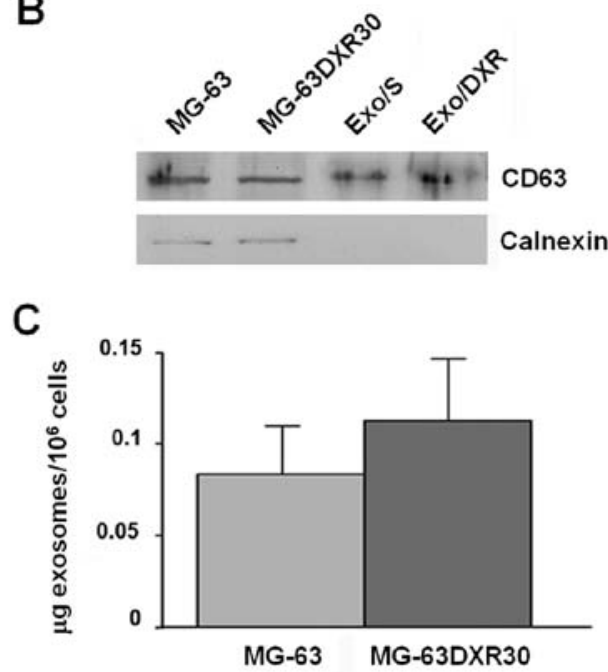

Figure 2. Characterization of exosomes. (A) Representative transmission electron microscopy image of MG-63 and MG-63DXR30 derived exosomes, showing a typical 'saucer-like' morphology and a diameter of 30-100 nm (scale bar, $100 \mathrm{~nm}$ ). (B) Exosome purity as assessed by western blot analysis for the expression of the specific exosomal marker CD63 and for endoplasmic reticulum protein calnexin. (C) Analysis of exosome concentration ( $\mu \mathrm{g}$ exosomes $/ 10^{6}$ cells) indicated similar quantities of exosomes secreted by MG-63 and MG-63DXR30 (mean $\pm \mathrm{SD}$; $\mathrm{n}=5$ ). Exo/S, exosomes derived from MG-63. Exo/DXR, exosomes derived from MG-63DXR30.

Reverse Transcriptase kit (Applied Biosystems, Thermo Fisher Scientific). The expression of MDR-1 (AF016535.1) was evaluated by quantitative real-time polymerase chain reaction (qRT-PCR) using the LightCycler instrumentation and the Universal Probe Library system (Roche Applied Science, Monza, Italy). Probes and primers were selected by a webbased assay design software (Probe Finder https://www. roche-applied-science.com): MDR-1-f 5'-GCCATCAGTCCT GTTCTTGG-3'; MDR-1-r 5'-GCTTTTGCATACGCTA AGAG TTC-3'. The results were expressed as the ratio between gene of interest and reference gene (GAPDH: NM_002046.3; GAPDH-f 5'-AGCCACATCGCTCAGACAC-3'; GAPDH-r 5 -GCCCAATACGACCAAATCC-3') according to the 2- ${ }^{\Delta \Delta C T}$ method (22).

Immunofluorescence assay.MG- 63 cells $\left(5 \times 10^{3} \mathrm{cells} / \mathrm{cm}^{2}\right)$ were seeded in IMDM + 10\% FDE. After $24 \mathrm{~h}$, cells were treated with $5 \mu \mathrm{g}$ of Exo/S or Exo/DXR or PBS (negative control) for $72 \mathrm{~h}$, and processed as previously described (23). At termination, cells were fixed in $3.7 \%$ PFA for $10 \mathrm{~min}$, permeabilized in $0.1 \%$ Triton X-100/PBS for 4 min, and blocked using 1\% BSA/ PBS for $30 \mathrm{~min}$. Cells were stained with an anti-MDR-1 antibody (sc-55510) (Santa Cruz Biotechnology) at 1:50 diluted in 
Actin
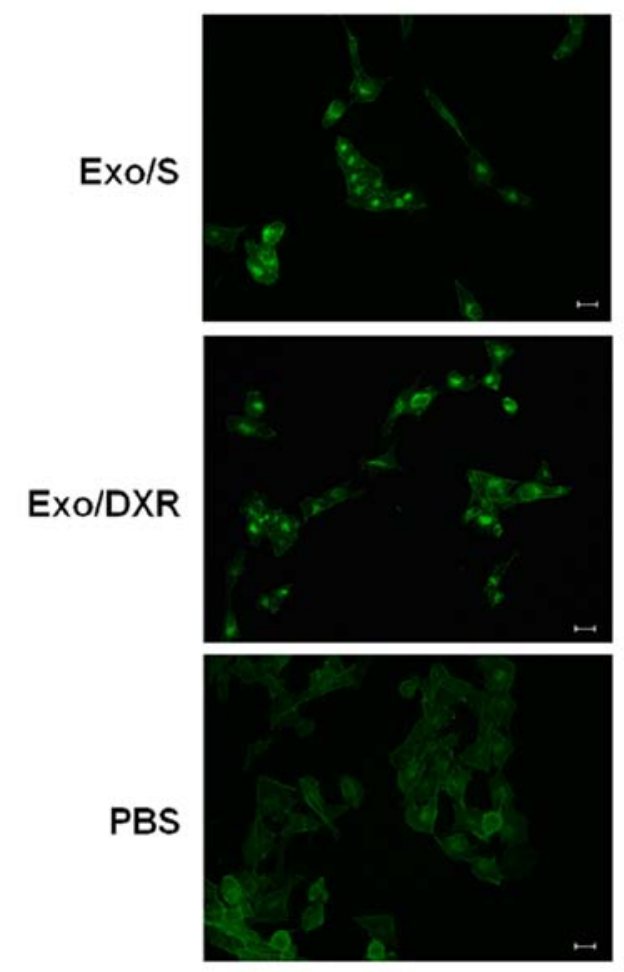

PKH26
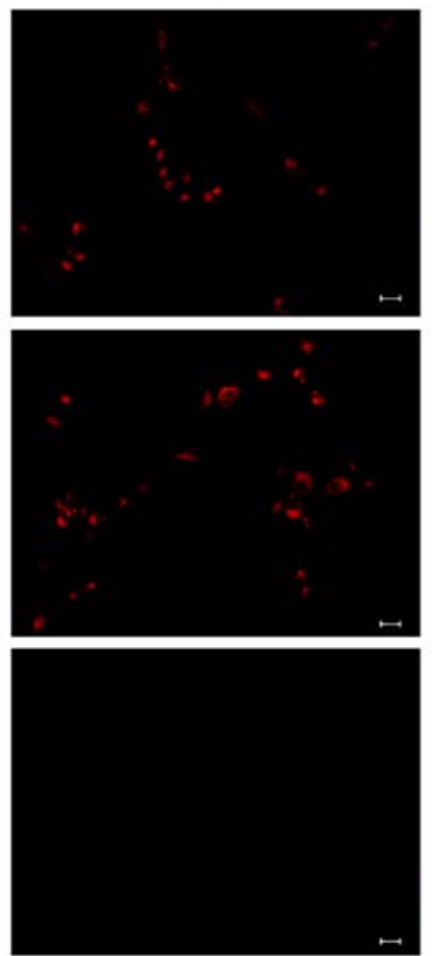

Merge
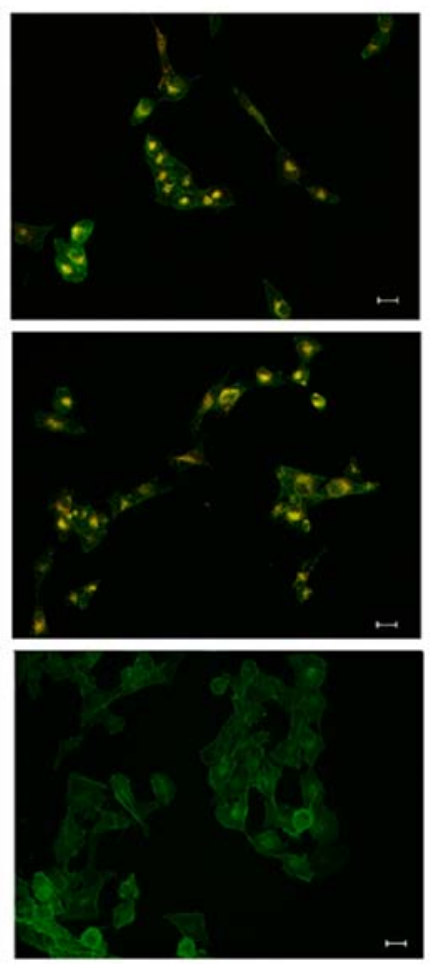

Figure 3. Uptake of exosomes by MG-63 cells. The uptake of the fluorescently labelled Exo/S and Exo/DXR (red) was evident in MG-63 cells after 24 h of incubation. No stain was revealed in the negative control condition (PBS). Actin filaments were stained with a FITC-conjugated phalloidin (green). Scale bar, $10 \mu \mathrm{m}$. Exo/S, exosomes derived from MG-63. Exo/DXR, exosomes derived from MG-63DXR30.

PBS containing $0.1 \%$ BSA at $22^{\circ} \mathrm{C}$ for $10-12 \mathrm{~h}$, followed by incubation with Alexa Fluor 568-conjugated goat anti-mouse secondary antibody (A11004) (Invitrogen) at 1:1,000 dilution in PBS containing $1 \% \mathrm{BSA}$ at $22^{\circ} \mathrm{C}$ for $30 \mathrm{~min}$. MG-63DXR30 cells were used as a positive control. Images were acquired with a Nikon Eclipse E800M fluorescence microscope (Nikon). Ten non-overlapping fields/image were taken (three images/sample were collected) and analysed using NIS-Elements Image software BR4.00.00 (Nikon). Changes of fluorescence intensity were calculated dividing the red fluorescent intensity by the number of cells on each field.

Statistical analysis. Statistical analysis was performed by StatView $^{\mathrm{TM}}$ 5.0.1 software (SAS Institute, Cary, NC, USA). Quantitative results were expressed as mean \pm the standard deviation. The Wilcoxon signed rank test was used to evaluate the effects of exosomes treatment on MDR-1/P-gp expression by real-time PCR and immunofluorescence, and to assess MG-63DXR30 effects on MG-63 viability by co-culture assays. The Mann-Whitney U test was applied to evaluate the quantity of exosome release and to analyze the effects of exosomes on cell viability and migration. Experiments were performed in triplicate. $\mathrm{p}<0.05$ was considered statistically significant.

\section{Results}

MG-63DXR30 cells transmit doxorubicin resistance to MG-63 cells. MG-63 were co-cultured with MG-63DXR30 in a 1:1 ratio, for $72 \mathrm{~h}$, in the presence of doxorubicin. As shown in Fig. 1, after drug exposure, survival of MG-63 cells was significantly increased $(\mathrm{p}=0.0039)$. These results suggested that MG-63DXR30 transmit chemoresistance to recipient cells, and such effect could be ascribed to exosomes.

Identification and characterization of exosomes. To investigate exosome correlation with resistance transmission, we collected exosomes from supernatant of MG-63 and MG-63DXR30 OS cell lines through a series of centrifugation and ultracentrifugation steps. Transmission electron microscopy analysis showed that the nanovesicles isolated from OS cells were morphologically homogeneous, ranging from 30 to $100 \mathrm{~nm}$ in size, with a typical round or cup shape appearance (Fig. 2A). Exosome purity was assessed by western blot analysis. As shown in Fig. 2B, they expressed exosome-related protein CD63 and, as expected, were negative for the endoplasmic reticulum protein calnexin (15). Ponceau S staining served as loading control (data not shown). Similar amounts of exosomes were secreted by MG-63 and MG-63DXR30 cells (Fig. 2C).

Uptake of exosomes. To examine whether exosomes from MG-63DXR30 (Exo/DXR) and MG-63 (Exo/S) could be taken up in MG-63 cells, PKH26 labeled exosomes were incubated with MG-63 cells at two different time points and examined using fluorescence microscopy. After $4 \mathrm{~h}$, a few exosomes were already taken up by MG-63 (data not shown). As shown in Fig. 3, after incubation, MG-63 cells were able to take up similar amount of Exo/DXR $(97.7 \pm 1.1 \%)$ and Exo/S 


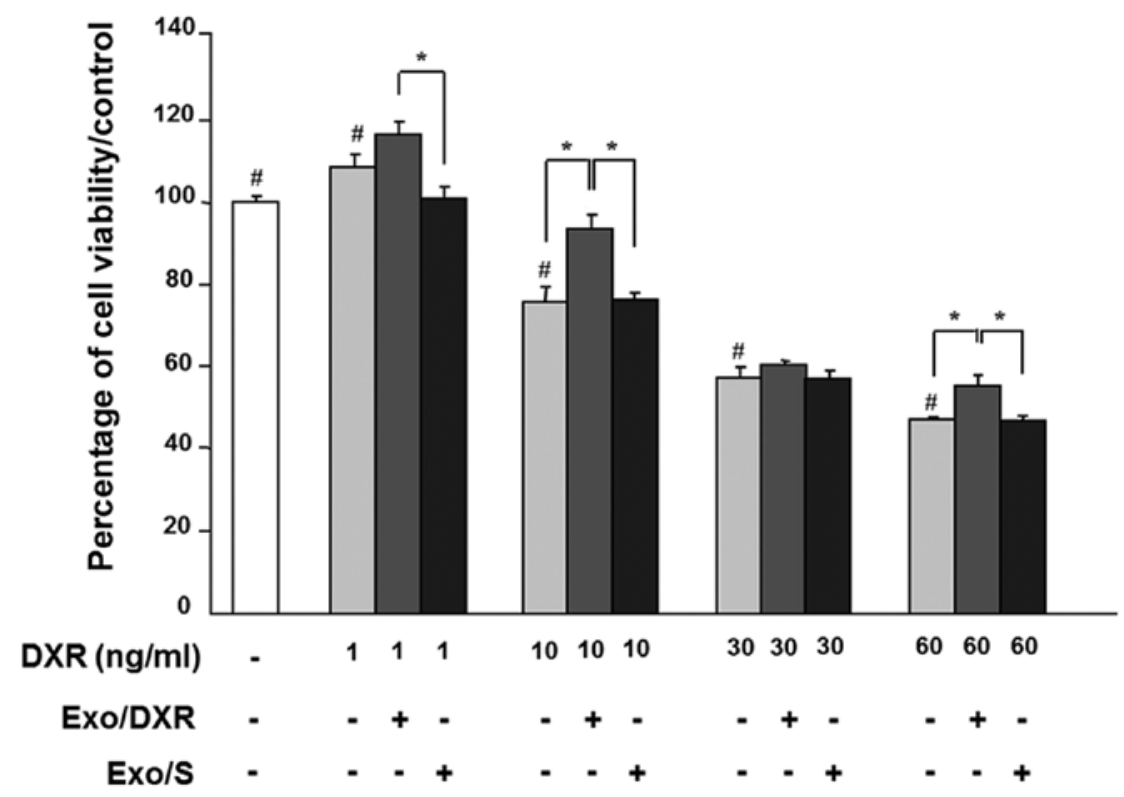

Figure 4. Effect of MG-63DXR30 derived exosomes on MG-63 sensitivity to doxorubicin. The sensitivity of MG-63 to doxorubicin is significantly lower in cells treated with Exo/DXR compared to untreated or Exo/S treated cells. Results represent triplicate biological repeats, each including three technical repeats and are displayed as mean $\pm \mathrm{SD}$, where " $\mathrm{p}<0.05$. Significant difference between MG-63 vs MG-63+DXR are shown as ${ }^{\#} \mathrm{p}<0.05$. Exo/S, exosomes derived from MG-63. Exo/DXR, exosomes derived from MG-63DXR30. DXR, doxorubicin.

A
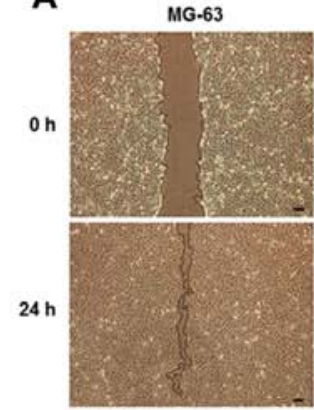

MG-63+ Exo/S

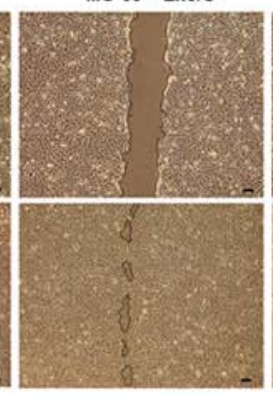

MG.63 + EXo/DXR

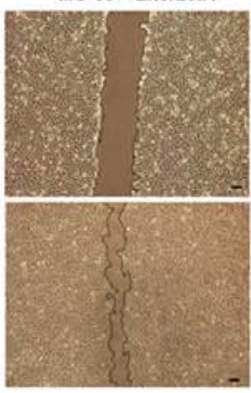

MG-63DXR30

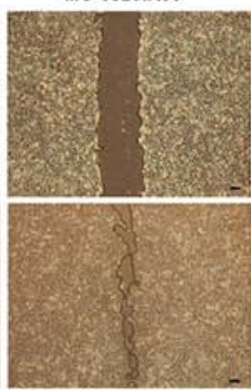

B

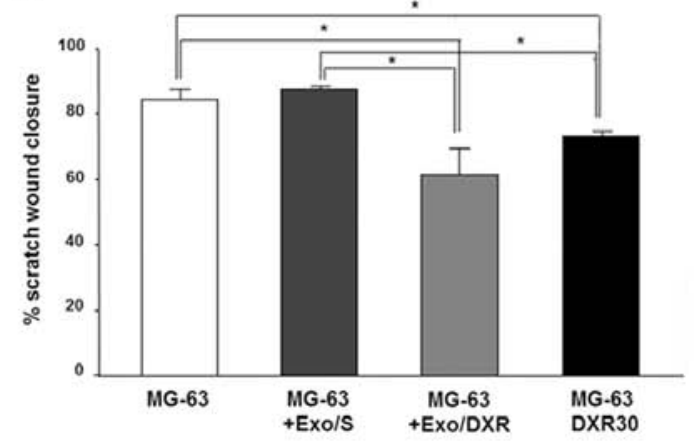

Figure 5. Effect of MG-63DXR30 derived exosomes on MG-63 cell motility. (A) Representative images of wound-healing assay. MG-63 and MG-63DXR30 monolayers were wounded with a $100-\mu \mathrm{l}$ pipette tip and images were taken by phase contrast microscopy immediately after scratch induction ( $0 \mathrm{~h})$ and $24 \mathrm{~h}$ after Exo/S or Exo/DXR treatment. Scale bar, $100 \mu \mathrm{m}$. (B) Quantification of the scratch wound-healing assay. The level of cell motility was quantified as the percentage of wound closure. Graph represents triplicate biological repeats and are displayed as mean $\pm \mathrm{SD}$, where * $<<0.05$. Exo/S, exosomes derived from MG-63. Exo/DXR, exosomes derived from MG-63DXR30.

$(97 \pm 1.4 \%)$. In particular, PKH26 signal was detected in the perinuclear region, suggesting the adsorption and internalization of exosomes. No fluorescent signal was detected in the control (PBS).

Exo/DXR decrease the sensitivity of MG-63 cells to doxorubicin. Cell viability was examined in exosome-treated MG-63 in the presence of increasing concentrations of doxorubicin (1-60 $\mathrm{ng} / \mathrm{ml}$ ) for $72 \mathrm{~h}$. As expected, doxorubicin affected MG-63 viability in a dose-dependent way. Incubation of MG-63 cells with $1.5 \mu \mathrm{g}$ of Exo/DXR decreased the sensitivity of cells to doxorubicin in all concentration tested. In particular, cells cultured at $10 \mathrm{ng} / \mathrm{ml}$ of doxorubicin and exposed to Exo/DXR showed a significant increase $(p=0.0095)$ in viability compared to cells incubated with doxorubicin alone
(Fig. 4). On the contrary, MG-63 viability was not modified when cells where treated with Exo/S (Fig. 4).

Exo/DXR decrease MG-63 cell motility. The effects of Exo/ DXR on MG-63 motility were evaluated by the woundhealing assay. After $24 \mathrm{~h}$ the migration of MG-63DXR30 was significantly lower than that of parental MG-63 cells $(\mathrm{p}=0.02)$. MG-63 treated with Exo/DXR exhibited a significant decrease of wound closure compared to MG-63 and MG-63 incubated with Exo/S ( $\mathrm{p}=0.02)$, whereas no difference was detected between Exo/DXR treated cells and MG-63DXR30 (Fig. 5A and B).

Exo/DXR expressed MDR-1 $m R N A$ and $P$-gp. To verify the presence of MDR-1 in exosome samples, we extracted total 
A

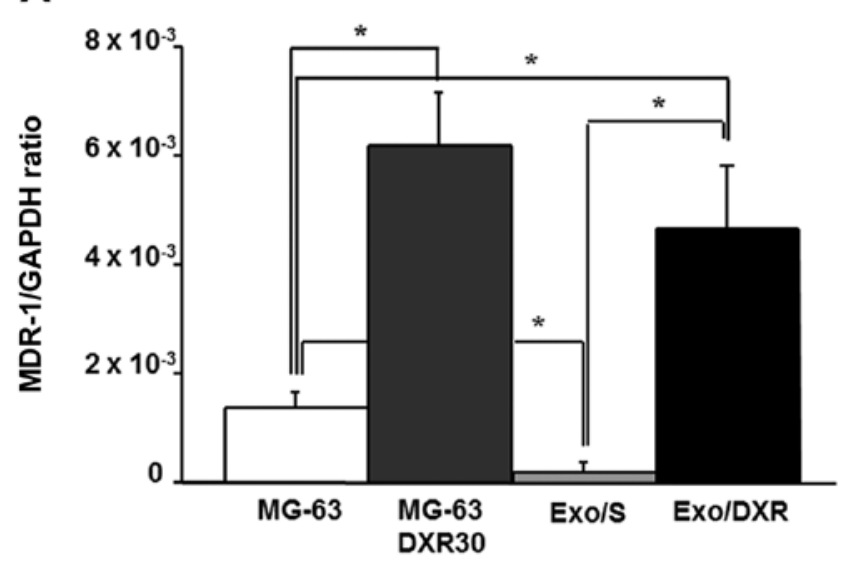

B

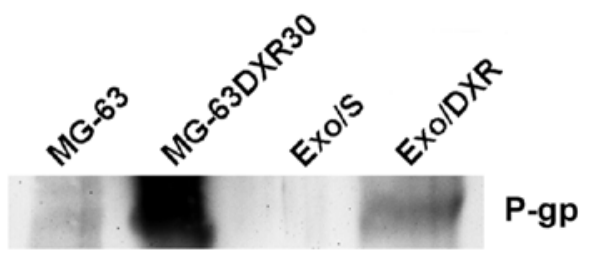

Figure 6. MDR-1 mRNA and P-gp expression in MG-63DXR30 derived exosomes. (A) Exosomes derived from MG-63DXR30 showed significantly higher level of MDR-1 mRNA expression compared to exosomes derived from MG-63 cells. MG-63DXR30, used as positive control, expressed substantially higher level of MDR-1 than MG-63. Analysis was carried out by qRT-PCR. Results represent triplicate biological repeats, each including three technical repeats and are displayed as mean $\pm \mathrm{SD}$, where ${ }^{*} \mathrm{p}<0.05$. (B) Western blot analysis of P-gp expression in MG-63DXR30 cells and their derived exosomes. A nearly undetectable P-gp protein expression was observed in MG-63 and Exo/S samples. Exo/S, exosomes derived from MG-63. Exo/DXR, exosomes derived from MG-63DXR30.

RNA from Exo/DXR, Exo/S and their cells of origin. The expression of MDR-1 was evaluated by qRT-PCR. Results demonstrated that Exo/DXR expressed higher levels of MDR-1 mRNA compared to Exo/S ( $\mathrm{p}=0.03$ ) (Fig. 6A). The western blot analysis was consistent with the results from qRT-PCR. Exo/DXR and their donor cells, but not MG-63 and Exo/S, showed high levels of P-gp expression by western blot analysis (Fig. 6B). Ponceau S staining served as loading control (data not shown).

Exo/DXR transfer MDR-1 mRNA and P-gp to recipient cells. The ability of Exo/DXR to transfer MDR-1 mRNA and P-gp was assessed by qRT-PCR and immunofluorescence analysis. Incubation of MG-63 cells with Exo/DXR induced a significant increase of MDR-1 mRNA levels compared to untreated cells $(p=0.02)$. On the contrary, no substantial change in MDR-1 expression was observed when MG- 63 cells were treated with Exo/S (Fig. 7A). As expected, MG-63DXR30 expressed significantly higher levels of MDR-1 mRNA compared to MG-63 cells $(\mathrm{p}=0.0006)$ (Fig. 7A). Immunofluorescence analysis confirmed a significant increase of P-gp expression in MG-63 treated with Exo/DXR ( $\mathrm{p}=0.009$ ) (Fig. 7B and C). Interestingly, MG-63 treated with Exo/DXR and MG-63DXR30 cells expressed similar levels of P-gp. The treatment with Exo/S did not affect P-gp protein levels (Fig. 7B and C).
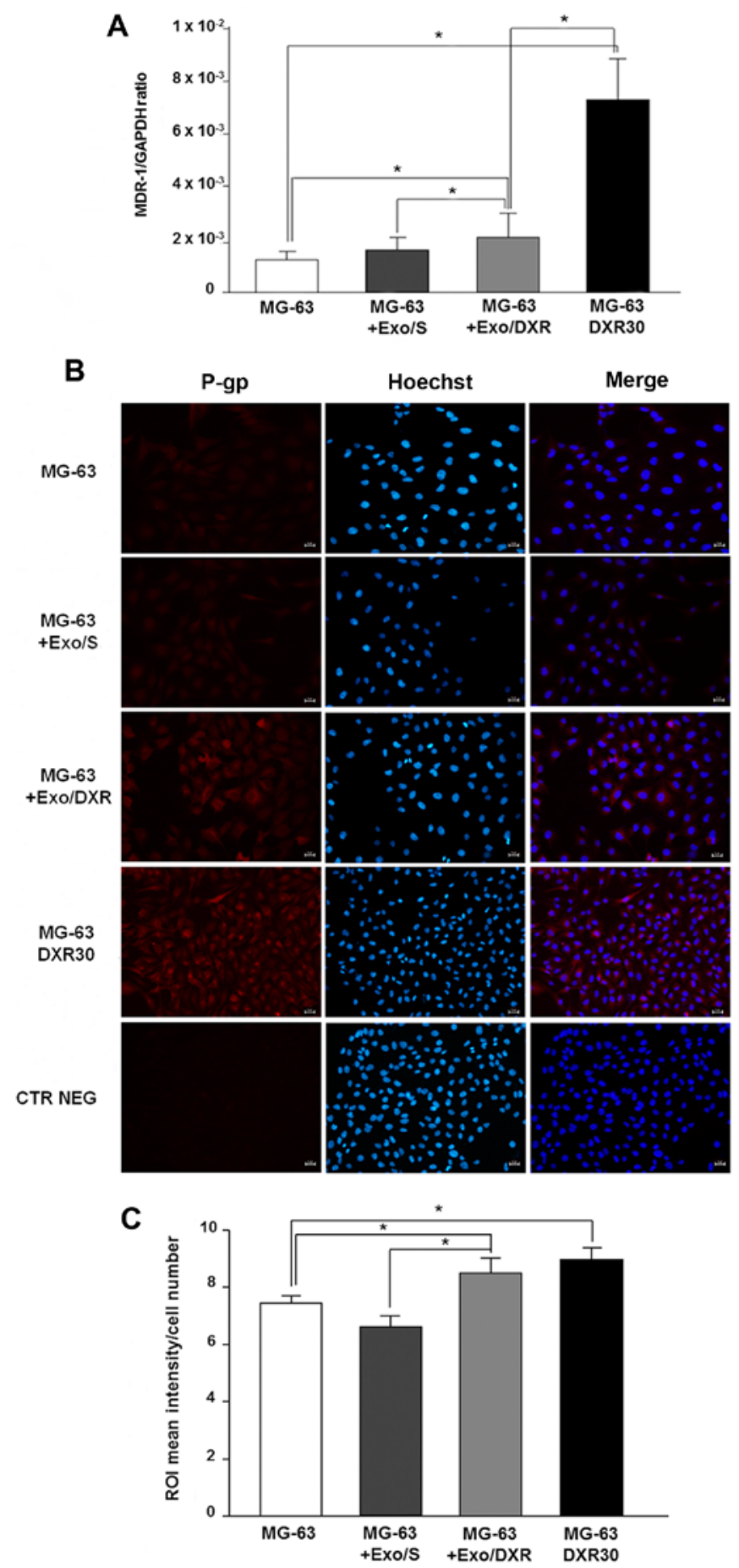

Figure 7. MDR-1 mRNA and P-gp expression in MG-63 cells following Exo/DXR treatment. (A) Following Exo/DXR treatment, MG-63 cells expressed significantly higher level of MDR-1 mRNA compared to MG-63 and MG-63 incubated with Exo/S. Analysis was carried out by qRT-PCR. Results represent triplicate biological repeats, each including three technical repeats and are displayed as mean $\pm \mathrm{SD}$, where ${ }^{*} \mathrm{p}<0.05$. (B) Representative images of immunofluorescence analysis of P-gp expression (red) in MG-63 cells treated with Exo/S or Exo/DXR. MG-63DXR30, used as positive control for P-gp expression. Negative staining control (CTR Neg) was performed by omitting the primary antibody. Nuclei were counterstained with Hoechst 33258 (blue). P-gp was clearly detected in MG-63 after incubation with Exo/DXR. Treatment with Exo/S did not influence P-gp expression. Scale bar, $10 \mu \mathrm{m}$. (C) Quantification of P-gp expression levels. P-gp expression of MG-63 significantly increased after Exo/DXR incubation, while the treatment with Exo/S did not affect P-gp protein levels. Similar levels of P-gp were detected between Exo/DXR treated cells and MG-63DXR30. Results are displayed as mean of red fluorescent intensity/number of cells $\pm \mathrm{SD}$, where ${ }^{*} \mathrm{p}<0.05$. Exo/S, exosomes derived from MG-63. Exo/DXR, exosomes derived from MG-63DXR30. 


\section{Discussion}

OS is the most common primary malignant bone tumour in children and adolescents (17). Although neoadjuvant chemotherapy and improved surgical techniques have increased the survival rate of OS $\leq 65-75 \%$, this is still unsuccessful in $30-40 \%$ of patients with localised tumours and in $80-85 \%$ of patients with metastatic disease at presentation $(24,25)$. MDR, both intrinsic and acquired, is still a major concern regarding the clinical management of OS patients and a key issue in the failure of current treatment (19). The MDR phenotype can be mediated by several mechanisms, including increased energydependent efflux of chemotherapeutic drugs (26). The principal transmembrane transporter responsible for this mechanism is P-glycoprotein (P-gp), a member of the ATP-binding cassette protein superfamily, encoded by the MDR-1 gene, which lowers intracellular drug concentrations to sub-lethal levels $(27,28)$. Although P-gp is involved in resistance or poor response to chemotherapy (29), other undefined cellular factors also seem to participate in modulating drug cytotoxicity. A growing body of evidence has demonstrated that several features of host microenvironment play also a role, including extracellular $\mathrm{pH}$, temperature, oxygen supply, and extracellular matrix (30-32). Moreover, the intercellular transfer of MDR represents an additional mechanism for the cellular acquisition and spreading of drug-resistant traits (33). Acquired MDR was recently found to be mediated by exosomes released by drug resistant cells (34). Such phenomenon was observed in several tumour models, including ovarian cancer (35), prostate cancer (14), breast cancer (15), and melanoma (36). In this study, we reported that MDR phenotype can also be induced in human OS via exosomes derived from MDR cells. MG-63DXR30 derived-exosomes are able to decrease MG-63 sensitivity to doxorubicin as well as to transfer phenotypic characteristics representative of their cell line of origin to recipient cells, including cell motility.

In agreement with previous studies $(13,15)$, we demonstrated that P-gp is contained in MG-63DXR30 derived-exosomes and that MDR can be transferred to sensitive cells by the delivery of P-gp in recipient cells through exosomes.

Moreover, exosomes are known to affect target cells by transferring mRNAs and microRNAs (37). The presence of mRNA and microRNA, termed 'exosomal shuttle RNA', in exosomes suggests that genetic material exchange could be an additional level of exosome-mediated intercellular communication (38). In particular, recent studies have described how the transfer of specific miRNA via exosomes potentially contributes to drug resistance in prostate cancer and breast cancer $(14,16)$.

In this study, we demonstrated, for the first time, that MDR-1 mRNA is highly expressed within Exo/DXR, and is transferred to and accumulated in OS sensitive cells after exosome treatment. The presence of selective MDR-1 mRNA in Exo/DXR suggests the intriguing possibility that this mRNA could be an additional factor that participate in drug resistance acquisition of sensitive cells.

In conclusion, this study corroborates the evidence that exosomes from MDR cells are capable of transferring chemoresistance by horizontal transfer of RNAs, including the specific mRNA of P-gp.

\section{Acknowledgements}

This study was supported by the Italian Ministry of Health, '5 per mille' 2012 grant awarded to Professor Nicola Baldini.

\section{References}

1. Théry C, Zitvogel L and Amigorena S: Exosomes: Composition, biogenesis and function. Nat Rev Immunol 2: 569-579, 2002.

2. Simons M and Raposo G: Exosomes - vesicular carriers for intercellular communication. Curr Opin Cell Biol 21: 575-581, 2009.

3. Raposo G, Nijman HW, Stoorvogel W, Liejendekker R, Harding CV, Melief CJ and Geuze HJ: B lymphocytes secrete antigen-presenting vesicles. J Exp Med 183: 1161-1172, 1996.

4. Zitvogel L, Regnault A, Lozier A, Wolfers J, Flament C, Tenza D, Ricciardi-Castagnoli P, Raposo G and Amigorena S: Eradication of established murine tumors using a novel cell-free vaccine: Dendritic cell-derived exosomes. Nat Med 4: 594-600, 1998.

5. Blanchard N, Lankar D, Faure F, Regnault A, Dumont C, Raposo G and Hivroz C: TCR activation of human T cells induces the production of exosomes bearing the TCR/CD3/zeta complex. J Immunol 168: 3235-3241, 2002.

6. Heijnen HF, Schiel AE, Fijnheer R, Geuze HJ and Sixma JJ: Activated platelets release two types of membrane vesicles: Microvesicles by surface shedding and exosomes derived from exocytosis of multivesicular bodies and alpha-granules. Blood 94: 3791-3799, 1999.

7. Fontana S, Saieva L, Taverna S and Alessandro R: Contribution of proteomics to understanding the role of tumor-derived exosomes in cancer progression: State of the art and new perspectives. Proteomics 13: 1581-1594, 2013.

8. De Jong OG, Van Balkom BW, Schiffelers RM, Bouten CV and Verhaar MC: Extracellular vesicles: potential roles in regenerative medicine. Front Immunol 5: 608, 2014.

9. Torreggiani E, Perut F, Roncuzzi L, Zini N, Baglìo SR and Baldini N: Exosomes: Novel effectors of human platelet lysate activity. Eur Cell Mater 28: 137-151, 2014.

10. Yang C and Robbins PD: The roles of tumor-derived exosomes in cancer pathogenesis. Clin Dev Immunol 2011: 842849, 2011.

11. Filipazzi P, Bürdek M, Villa A, Rivoltini L and Huber V: Recent advances on the role of tumor exosomes in immunosuppression and disease progression. Semin Cancer Biol 22: 342-349, 2012.

12. Gong J, Jaiswal R, Mathys JM, Combes V, Grau GE and Bebawy M: Microparticles and their emerging role in cancer multidrug resistance. Cancer Treat Rev 38: 226-234, 2012.

13. Corcoran C, Rani S, O'Brien K, O'Neill A, Prencipe M, Sheikh R, Webb G, McDermott R, Watson W, Crown J, et al: Docetaxelresistance in prostate cancer: Evaluating associated phenotypic changes and potential for resistance transfer via exosomes. PLoS One 7: e50999, 2012.

14. Corcoran C, Rani S and O'Driscoll L: miR-34a is an intracellular and exosomal predictive biomarker for response to docetaxel with clinical relevance to prostate cancer progression. Prostate 74: 1320-1334, 2014.

15. Lv MM, Zhu XY, Chen WX, Zhong SL, Hu Q, Ma TF, Zhang J, Chen L, Tang JH and Zhao JH: Exosomes mediate drug resistance transfer in MCF-7 breast cancer cells and a probable mechanism is delivery of P-glycoprotein. Tumour Biol 35: 10773-10779, 2014.

16. Chen WX, Liu XM, Lv MM, Chen L, Zhao JH, Zhong SL, Ji MH, $\mathrm{Hu} \mathrm{Q}$, Luo Z, Wu JZ, et al: Exosomes from drug-resistant breast cancer cells transmit chemoresistance by a horizontal transfer of microRNAs. PLoS One 9: e95240, 2014.

17. Heare T, Hensley MA and Dell'Orfano S: Bone tumors: Osteosarcoma and Ewing's sarcoma. Curr Opin Pediatr 21: 365-372, 2009.

18. Baldini N, Scotlandi K, Barbanti-Bròdano G, Manara MC, Maurici D, Bacci G, Bertoni F, Picci P, Sottili S, Campanacci M, et al: Expression of P-glycoprotein in high-grade osteosarcomas in relation to clinical outcome. N Engl J Med 333: 1380-1385, 1995.

19. Li S, Sun W, Wang H, Zuo D, Hua Y and Cai Z: Research progress on the multidrug resistance mechanisms of osteosarcoma chemotherapy and reversal. Tumour Biol 36: 1329-1338, 2015.

20. Roncuzzi L, Pancotti F and Baldini N: Involvement of HIF-1a activation in the doxorubicin resistance of human osteosarcoma cells. Oncol Rep 32: 389-394, 2014. 
21. Salerno M, Avnet S, Bonuccelli G, Hosogi S, Granchi D and Baldini N: Impairment of lysosomal activity as a therapeutic modality targeting cancer stem cells of embryonal rhabdomyosarcoma cell line RD. PLoS One 9: e110340, 2014

22. Livak KJ and Schmittgen TD: Analysis of relative gene expression data using real-time quantitative PCR and the $2-{ }^{\Delta \Delta C T}$ method. Methods 25: 402-408, 2001.

23. Su L, Mruk DD, Lui WY, Lee WM and Cheng CY: P-glycoprotein regulates blood-testis barrier dynamics via its effects on the occludin/zonula occludens 1 (ZO-1) protein complex mediated by focal adhesion kinase (FAK). Proc Natl Acad Sci USA 108 : 19623-19628, 2011.

24. Mankin HJ, Hornicek FJ, Rosenberg AE, Harmon DC and Gebhardt MC: Survival data for 648 patients with osteosarcoma treated at one institution. Clin Orthop Relat Res 429: 286-291, 2004.

25. Bacci G, Briccoli A, Rocca M, Ferrari S, Donati D, Longhi A, Bertoni F, Bacchini P, Giacomini S, Forni C, et al: Neoadjuvant chemotherapy for osteosarcoma of the extremities with metastases at presentation: Recent experience at the Rizzoli Institute in 57 patients treated with cisplatin, doxorubicin, and a high dose of methotrexate and ifosfamide. Ann Oncol 14: 1126-1134, 2003.

26. Gottesman MM, Fojo T and Bates SE: Multidrug resistance in cancer: Role of ATP-dependent transporters. Nat Rev Cancer 2: 48-58, 2002.

27. Borst $\mathrm{P}$ and Elferink RO: Mammalian $\mathrm{ABC}$ transporters in health and disease. Annu Rev Biochem 71: 537-592, 2002.

28. Ambudkar SV, Kimchi-Sarfaty C, Sauna ZE and Gottesman MM: P-glycoprotein: From genomics to mechanism. Oncogene 22: 7468-7485, 2003.

29. Baldini N, Scotlandi K, Serra M, Picci P, Bacci G, Sottili S and Campanacci M: P-glycoprotein expression in osteosarcoma: A basis for risk-adapted adjuvant chemotherapy. J Orthop Res 17: 629-632, 1999

30. Harguindey S, Orive G, Luis Pedraz J, Paradiso A and Reshkin SJ The role of $\mathrm{pH}$ dynamics and the $\mathrm{Na}^{+} / \mathrm{H}^{+}$antiporter in the etiopathogenesis and treatment of cancer. Two faces of the same coin - one single nature. Biochim Biophys Acta 1756: 1-24, 2005.
31. Shicang Y, Guijun H, Guisheng Q, Yuying L, Guoming W and Ruiling G: Efficacy of chemotherapeutic agents under hypoxic conditions in pulmonary adenocarcinoma multidrug resistant cell line. J Chemother 19: 203-211, 2007.

32. Chen KG and Sikic BI: Molecular pathways: Regulation and therapeutic implications of multidrug resistance. Clin Cancer Res 18: 1863-1869, 2012.

33. Levchenko A, Mehta BM, Niu X, Kang G, Villafania L, Way D, Polycarpe D, Sadelain M and Larson SM: Intercellular transfer of P-glycoprotein mediates acquired multidrug resistance in tumor cells. Proc Natl Acad Sci USA 102: 1933-1938, 2005.

34. Zhao L, Liu W, Xiao J and Cao B: The role of exosomes and 'exosomal shuttle microRNA' in tumorigenesis and drug resistance. Cancer Lett 356B: 339-346, 2015.

35. Safaei R, Larson BJ, Cheng TC, Gibson MA, Otani S, Naerdemann W and Howell SB: Abnormal lysosomal trafficking and enhanced exosomal export of cisplatin in drug-resistant human ovarian carcinoma cells. Mol Cancer Ther 4: 1595-1604, 2005.

36. Federici C, Petrucci F, Caimi S, Cesolini A, Logozzi M, Borghi M, D'Ilio S, Lugini L, Violante N, Azzarito T, et al: Exosome release and low $\mathrm{pH}$ belong to a framework of resistance of human melanoma cells to cisplatin. PLoS One 9: e88193, 2014

37. Valadi H, Ekström K, Bossios A, Sjöstrand M, Lee JJ and Lötvall JO: Exosome-mediated transfer of mRNAs and microRNAs is a novel mechanism of genetic exchange between cells. Nat Cell Biol 9: 654-659, 2007.

38. Ramachandran $\mathrm{S}$ and Palanisamy V: Horizontal transfer of RNAs: Exosomes as mediators of intercellular communication. Wiley Interdiscip Rev RNA 3: 286-293, 2012. 